OPEN ACCESS

Edited by:

Barbara Seitz-Polski,

University of Nice Sophia Antipolis,

France

Reviewed by:

Alfred Hyoungju Kim,

Washington University School of

Medicine in St. Louis, United States

*Correspondence:

Fabian Braun

fa.braun@uke.de

${ }^{\dagger}$ These authors have contributed equally to this work

Specialty section:

This article was submitted to Autoimmune and Autoinflammatory

Disorders,

a section of the journal

Frontiers in Immunology

Received: 22 October 2021 Accepted: 20 December 2021

Published: 13 January 2022

Citation:

Muehlig AK, Gies $S$, Huber TB and Braun F (2022)

Collapsing Focal Segmental

Glomerulosclerosis

in Viral Infections.

Front. Immunol. 12:800074. doi: 10.3389/fimmu.2021.800074

\section{Collapsing Focal Segmental Glomerulosclerosis in Viral Infections}

\author{
Anne K. Muehlig ${ }^{1,2,3 \dagger}$, Sydney Gies ${ }^{1 \dagger}$, Tobias B. Huber ${ }^{1}$ and Fabian Braun ${ }^{1 *}$ \\ ${ }^{1}$ III. Department of Medicine, University Medical Center Hamburg-Eppendorf, Hamburg, Germany, ${ }^{2}$ University Children's \\ Hospital, University Medical Center Hamburg-Eppendorf, Hamburg, Germany, ${ }^{3}$ University Children's Research@Kinder-UKE, \\ University Medical Center Hamburg-Eppendorf, Hamburg, Germany
}

Collapsing glomerulopathy represents a special variant of the proteinuric kidney disease focal segmental glomerulosclerosis (FSGS). Histologically, the collapsing form of FSGS (cFSGS) is characterized by segmental or global condensation and obliteration of glomerular capillaries, the appearance of hyperplastic and hypertrophic podocytes and severe tubulointerstitial damage. Clinically, cFSGS patients present with acute kidney injury, nephrotic-range proteinuria and are at a high risk of rapid progression to irreversible kidney failure. cFSGS can be attributed to numerous etiologies, namely, viral infections like HIV, cytomegalovirus, Epstein-Barr-Virus, and parvovirus B19 and also drugs and severe ischemia. Risk variants of the APOL1 gene, predominantly found in people of African descent, increase the risk of developing cFSGS. Patients infected with the new CoronaVirus SARS-CoV-2 display an increased rate of acute kidney injury (AKI) in severe cases of COVID-19. Besides hemodynamic instability, cytokine mediated injury and direct viral entry and infection of renal epithelial cells contributing to AKI, there are emerging reports of cFSGS associated with SARS-CoV-2 infection in patients of mainly African ethnicity. The pathogenesis of CFSGS is proposed to be linked with direct viral infection of podocytes, as described for HIV-associated glomerulopathy. Nevertheless, there is growing evidence that the systemic inflammatory cascade, activated in acute viral infections like COVID-19, is a major contributor to the impairment of basic cellular functions in podocytes. This mini review will summarize the current knowledge on cFSGS associated with viral infections with a special focus on the influence of systemic immune responses and potential mechanisms propagating the development of cFSGS.

Keywords: podocyte, APOL 1, HIVAN, COVAN, Immune response

\section{INTRODUCTION}

Focal segmental glomerulosclerosis (FSGS) is a major glomerular cause of end stage renal disease. The definition of FSGS is derived from its histopathological picture-the focal appearance of segmental scarring in some glomeruli. Before sclerosis ensues, podocytes show foot process effacement, leading to the manifestation of proteinuria. Since FSGS represents a pattern of response to injury, it was recently proposed to group FSGS into primary (immune-mediated) FSGS, adaptive FSGS, FSGS caused by pregnancy-related VEGF-inhibition, genetic, drug- and virus-associated FSGS $(1,2)$. The Columbia 
classification of FSGS differentiates 5 morphologic variants, namely, FSGS not otherwise specified (NOS), perihilar, cellular, tip, and collapsing variant (3).

The collapsing form of FSGS (cFSGS) represents a special form of secondary FSGS. Histopathologically, cFSGS is characterized by segmental or global condensation and obliteration of the capillary tuft associated with wrinkling and collapsing of the glomerular basement membrane. The podocytes display a distinct hyperplastic and hypertrophic phenotype, often containing cytoplasmic protein resorption droplets and pronounced foot process effacement. Severe tubulointerstitial disease with inflammation, edema, interstitial fibrosis and tubular atrophy as well as tubular regenerative changes constitutes an important component of cFSGS (4). cFSGS is associated with different etiologies. One of the best characterized causes is an infection with the human immune deficiency virus (HIV) and the development of HIV-associated nephropathy (HIVAN) (5). Furthermore, cFSGS can also be attributed to other infections, drugs, severe ischemia, autoimmune disease, genetic causes, and idiopathic (6-9). Additionally, an infection with the new Corona-Virus during the pandemic of SARS-CoV2 has been associated with the potential to develop cFSGS.

\section{HIV-ASSOCIATED cFSGS}

HIV infection accompanied by acute kidney injury, proteinuria, and a rapid progression to irreversible kidney failure characterizes the course of HIVAN. Tubuloreticular aggregates in endothelial cells and microcystic tubular dilatation in some cases may contribute to differentiate HIVAN from other etiologies of cFSGS in light microscopy (7).

Investigating the interaction of the virus or viral gene products with podocyte signaling pathways that induce massive morphologic alterations in cFSGS, might contribute to our understanding of podocyte biology and find a targeted therapy in (collapsing) FSGS independent of its etiology. It has been shown, even though podocytes do not express CD4-receptors or other known HIVreceptors, that podocytes are directly infected by HIV (10). The virus is known to damage the actin cytoskeleton in any cell type (11). The podocyte cytoskeleton is essential for the maintenance of the glomerular filtration barrier.

Furthermore, it has been shown, that HIV-1 also induces vascular endothelial growth factor (VEGF), leading to proliferation and de-differentiation of podocytes in cFSGS (12). Podocyte VEGF overexpression in a mouse model was able to cause glomerular disease with podocyte foot process effacement (13), while it was also shown that VEGF is crucial for podocyte survival via phospatidyl inositol 3 kinase/Akt signaling (14).

HIV associated cFSGS predominantly affects patients of African descent carrying a risk variant of the Apolipoprotein $\mathrm{L}$ gene 1 (APOL1), termed G1 and G2. The only known physiological function of APOL1 is its anti-trypanosomal activity (15). Several subspecies of trypanosoma have developed resistency against the "normal" G0 variants of APOL1. Therefore, the presence of one of the APOL1 variants G1 or G2 appears to protect against infection of several subspecies of Trypanosoma brucei (16-18). APOL1 is an abundantly secreted protein that circulates and associates with apolipoprotein A-I as a component of high-density lipoprotein (HDL) (19). It is also expressed in the intracellular compartment of various tissues-in the kidney specifically in glomeruli, proximal tubular endothelia and arteriolar endothelium. Within healthy glomeruli, APOL1 is localized exclusively in the podocytes (20). In both FSGS and HIVAN however, APOL1 expressing $\alpha$-smooth muscle actin-positive cells were detected in the media of medium arteries and arterioles.

The APOL1 risk alleles G1 and G2 increase the risk of chronic kidney disease and are associated with an elevated risk of developing hypertension-associated end stage renal disease, FSGS, Lupus-nephritis, and HIVAN (16, 21-23).

Numerous studies suggested multiple pathways leading to impaired podocyte function and injury in HIVAN. The lack of the APOL1 gene in most model organisms and the absence of tissue specificity constitute barriers in identifying the underlying mechanisms. Conversely, the lack of the APOL1 gene in most mammals and a case report of a healthy APOL1-null patient supports the hypothesis, that APOL1 is not essential for kidney development and homeostasis (24).

The overexpression of APOL1 risk variants in podocytes was associated with increased necrosis and lysosomal permeability with leakage of lysosomal enzymes like cathepsin $\mathrm{L}$ into the cytosolic and nuclear compartment. Cathepsin L-induced degradation of the cytoskeletal protein F-actin might contribute to podocyte injury (25). Reduced numbers of autolysosomes led to impaired autophagic flux in APOL1 risk variant expressing HEK293 cells and podocytes (26). APOL1 risk variant dependent on upregulation of miR193a was found to result in the dedifferentiation of podocytes by blocking autophagy (27). Furthermore, APOL1 risk variants seem to downregulate expression levels of nephrin and podocin, key players in the slit diaphragm, and mediators of crucial signaling pathways (28).

These studies indicated the important role of APOL1 in the development of FSGS, as podocyte loss due to cell death occurred in all models of APOL1 risk variant overexpression. Pyroptosis, but not apoptosis was found to be increased in APOL1 risk allele transfected cells and might be a result of elevated levels of cleaved caspase 1 (26).

Scientific efforts could show that expression of human APOL1 risk variants in kidneys, spleens, and macrophages of bacterial artificial chromosome (BAC) transgenic mice promotes cholesterol accumulation (29). BAC/APOL1 transgenic mice express either the G0 allele or the risk alleles G1 and G2 under the endogenous APOL1 promotor. In line with these results, a recent study demonstrated in an APOL1/BAC mouse model that APOL1 risk variant expression drives lipid accumulation in renal cortices but not proteinuria. APOL1 risk variant expression in transgenic mice of a FSGS model (APOL1; Podocin-rtTA; NFATc1 ${ }^{\text {nuc }}$ ) were more susceptible to doxycyclin induction as their wildtype littermates (WT; Podocin-rtTA; NFATc1 ${ }^{\text {nuc }}$ ) and developed podocyte loss and mesangial matrix expansion. Interestingly, at baseline the human APOL1 transgenic mice did not develop proteinuria. The investigation of urinary podocytes from FSGS patients carrying either the G0/G0 or G1/G2 allele 
suggested that the APOL1 risk variants cause mitochondrial dysfunction linked with lipid accumulation and compensatory OXPHOS complexes elevation in podocytes (30).

Since most carriers of two risk alleles do not develop kidney disease spontaneously and the APOL1 variant expression itself was not sufficient to induce podocyte dysfunction, a second hit is postulated essential for the development of kidney disease. Infection with $\mathrm{HIV}$ is believed to be the strongest known risk factor for APOL1-associated kidney disease (18) as the innate immune response to HIV upregulates APOL1 gene expression (31).

Several innate immune pathways like interleukin $1 ß$ and Tolllike receptor 3 (TLR3) signaling showed impact on APOL1 expression levels, but the predominant effect upon HIV infection is demonstrated for interferons (INF), especially for type 1 INF (INF- $\alpha$ and - $\beta$ ) (32-34). Type 1 INF represents a part of the innate host response against viruses. It induces the intracellular response to viral infection by orchestrating a signaling cascade through the Janus kinase signal transducer and activator of transcription (JAKSTAT) pathway. The JAK-STAT-pathway regulates the transcription of INF-regulated genes (IRG), that contribute to reduce viral spread via distinct mechanism like the inhibition of virus entry, translation, replication and viral egress (35).

Additionally, type $1 \mathrm{INF}$ induces apoptosis of infected cells in an autocrine and paracrine manner. Acute and chronic HIV infections constitute a proinflammatory state with elevated levels of interferon in plasma and tissue (36). It was indicated that type 1 INF- $\alpha$ and $-\beta$ and also type 2 INF $\gamma$ is able to upregulate APOL1 gene expression in both endothelial cells and podocytes (34). In addition, case reports describe patients carrying APOL1 risk variants who developed cFSGS after treatment with exogenous INF $(34,37)$ and INF- $\gamma$ induced proteinuria in APOL1 G1 transgenic mice (38).

Stimulator of interferon genes (STING) is suggested to be an INF-induced candidate pathway that upregulates APOL1. HIV infection induces cyclic guanosine monophosphate-adenosine (cGAMP) synthase (cGAS) to produce cGAMP, an activator of STING (39). Interferon-inducible protein 16 (IFI16) has also been shown to act as a sensor of HIV infection and activator of STING (40). TANK binding kinase 1 (TBK1) is recruited by activated STING and phosphorylates STING and interferon regulatory factor 3 (IRF3). Phosphorylated IRF3 is then translocated to the nucleus and initiates transcription of INF- $\beta$ and APOL1 in human podocytes. INF- $\beta$ can furthermore activate the type I IFN receptor, leading to STAT1 phosphorylation through IFNARassociated JAK1 kinase and increased upregulation of APOL1 and IFI16, further enhancing upregulation of STING (41). Further, cGAS/STING seem to play a key role in the increased endothelial dysfunction mediated by APOL1 risk variants and might contribute to explain the increased sepsis incidence and severity among patients of African ancestry (42).

Both cGAS and IFI16 might also play a role in the progression to lupus nephritis in SLE patients carrying risk alleles of APOL1 $(41,43)$.

In line with these results, it was demonstrated that retinoic acid-inducible gene I (RIG-I) also recognizes HIV and enhances APOL1 expression and also activation of nuclear factor kappa B (NFKB). The knockdown of RIG-I in human podocytes resulted in attenuated inflammatory and apoptotic effects of APOL1 (44).
Additionally, TLR 3 signaling, that can be activated by double stranded RNAs, can be found in replication cycles of nearly all viruses and was shown to enhance APOL1 expression INFindependent via NFKB-signaling (34).

To summarize, the described interactions lead to an enhanced expression of APOL1. As the risk variants seem to exert an endotoxic effect on podocytes in a dose-dependent manner, this overexpression can induce podocyte damage in case of HIV infection, whereas, the toxic influence of the risk variants might not be high enough to induce cell damage and therefore cFSGS, without the "second hit" HIV.

Another recently debated mechanism is the contribution of parietal epithelial cells (PEC) to the cFSGS. PECs are capable of self-renewal and differentiation into various cell types, namely, podocytes (45-47) while the regeneration of podocytes appears limited to non-existent $(47,48)$. It has been shown in vitro, that the induction of APOL1 in PECs leads to the expression of podocyte markers, potentially as a repair mechanism and the effort to replace damaged podocytes (49). Furthermore, analysis of the glomerular extracellular matrix shows differences between non-collapsing and cFSGS, with an altered extracellular matrix remodelling and activation of PECs (50).

PEC activation can be detected as a first sign of ensuing glomerular scarring (51). Podocyte hypertrophy, as present in cFSGS, seems to prevent PEC activation and glomerulosclerosis. In glomerular extracts from biopsies of FSGS and diabetic nephropathy mammalian target of rapamycin (mTOR) and PEC-activation related genes were found to be upregulated (48). mTOR-mediated podocyte hypertrophy during podocyte loss seems to be crucial to maintain glomerular functional integrity, as pharmacological mTOR-inhibition during acute podocyte loss resulted in albuminuria, PEC-activation and glomerulosclerosis in mice (48). Interestingly, exacerbated and persistent podocyte hypertrophy also induced podocyte loss and PEC-activation, indicating a limited beneficial effect (48).

These data suggest the targeting of PECs as a potential therapeutic option in cFSGS. APOL1-risk variant effects on podocytes might impair their capacity to prevent PEC-activation and glomerulosclerosis.

\section{COVID-ASSOCIATED NEPHROPATHY (COVAN)}

Renal involvement with acute kidney injury (AKI), proteinuria and hematuria, worsening the overall prognosis, has been shown frequently in COVID-19 patients (52-54). Chronic kidney disease or conditions with increased risk of impaired kidney function represent strong risk factors for a severe clinical course (55). Biopsy and autopsy studies revealed acute tubular necrosis (ATN) in the majority of COVID-19 associated AKI. Nevertheless, glomerular involvement was also reported and should be distinguished from the majority of AKI due to ATN. Beside reports of minimal change disease, membranous nephropathy, anti-glomerular basement membrane glomerulonephritis, IgA-vasculitis, lupus nephritis and crescentic glomerulonephritis (56-58) associated with SARS-CoV-2, 
cases of cFSGS displaying similar lesions, observed in HIVAN, have led to the emergence of the SARS-CoV-2 associated entity named "COVAN" (59). COVAN patients mostly present with severe AKI and nephrotic range proteinuria in native kidneys and kidney allografts (60-62).

Like HIVAN, COVAN is mostly reported in patients carrying risk variants of APOL1 supporting the hypothesis of a "second hit" necessary for the onset of APOL1-associated kidney disease. Paying tribute to the short time period of research on COVAN, compared to decades of HIV-research, only few mechanistic data are available for SARS-CoV-2 induced cFSGS. Interestingly, although intracellular and not secreted APOL1 seems to be responsible for kidney injury, a case of SARS-CoV-2 associated cFSGS was reported in a patient carrying 2 APOL1 high risk alleles with a kidney allograft stemming from a low-risk genotype donor (62).

Cytokine induced podocyte damage by the virus and/or direct toxic viral effects on podocytes are suggested to be responsible for SARS-CoV-2 associated cFSGS and might interact with APOL1. The membrane proteins angiotensin converting enzyme II (ACE2) and transmembrane serin protease 2 (TMPRSS2) are used as receptors by SARS-CoV-2 to facilitate cell entry. ACE2 is highly expressed in kidney cells, mainly in the proximal tubule. However, podocytes, parietal epithelial cells, mesangial cells and cells of the collecting duct are found to express ACE2 at lower levels (63). Autopsy studies could detect SARS-CoV-2 RNA and viral proteins in the kidney $(64,65)$ and indicated, that SARS-CoV-2 renal tropism is associated with the development of acute kidney injury and disease severity (66). In contrast, cases of cFSGS occurred also in patients with mild or even absent respiratory symptoms (67). To our knowledge, all biopsy studies failed to detect SARS-CoV-2 in the kidney. This limits investigating direct toxic viral effects on podocytes and implicates cytokine-mediated effects as shown for HIVAN. Nevertheless, most studies relied on electron microscopy, immunohistochemistry or RNA in situ hybridization limiting the conclusions drawn concerning viral detection. PCR based detection methods were regularly able to detect viral RNA within kidney tissue and complimentary RNA and protein detection underline a probable renal SARS-CoV-2 tropism $(64,65,68-70)$.

Next to the direct infection of kidney tissue, an injury of the glomerulus induced by the host response might be another plausible mechanism of the development of cFSGS in COVID-19. The often described "cytokine storm" can damage the kidney directly or secondary through the induction of life-threatening circumstances like sepsis, shock, ischemia, hypoxia or rhabdomyolysis (71). Especially patients with severe disease show high plasma levels of cytokines like interleukins, granulocyte cell stimulating factor and tumor necrosis factor $\alpha$ (TNF $\alpha)$ (72). Interferon $\gamma$ (INF $\gamma$ ) is also upregulated in SARS-CoV-2 infected patients, but a meta-analysis could not show significant differences between the severe and nonsevere group of COVID-19 patients (73). Nevertheless, data from subgroups carrying APOL1 risk alleles are not available to our knowledge. A recent study observed that APOL1 risk variants are associated with a higher incidence of sepsis and increased disease severity in patients with COVID-19. Plasma levels of APOL1 were higher in patients with severe sepsis and COVID-19 and correlated with markers of endothelial dysfunction. A mouse model with endothelial cell specific expression of APOL1 risk alleles, developed increased endothelial inflammation, vascular leakage, albuminuria and increased sepsis severity. APOL1 risk variant expression in endothelial cells in vitro resulted in mitophagy and leakage of mitochondrial DNA into cytoplasm. Cytosolic DNA is sensed by the NLRP3 inflammasome and by cGAS, an activator of STING, leading to endothelial dysfunction (42). The onset and role of endothelial dysfunction in cFSGS is poorly understood. Although it is commonly assumed that podocyte injury is the first event in the pathogenesis of cFSGS, it was demonstrated, that in Adriamycininduced nephropathy, endothelial damage occurs prior to podocyte injury (74). Additionally, it was shown that patients with primary FSGS and nephrotic range proteinuria had elevated markers of endothelial dysfunction compared to healthy controls, which were largely related to the activity of the disease (75).

Beyond these recent observations, upregulated cytokines in COVID-19 patients carrying risk alleles of APOL1 could still contribute to the development of cFSGS as a "second hit" as the expression of chemokines (e.g., CCL2 and CXCL10) and Interleukin 6 seems to be elevated within the kidney of COVID 19 patients (76).

It remains elusive, whether podocytes or endothelial cells predominantly orchestrate the histopatholgical changes. All known mechanisms, which lead to kidney damage beyond cFSGS in case of infection with SARS-CoV-2 have been extensively reviewed by Ahmadian et al. (77).

\section{cFSGS and Other Viral Infections}

While the association of cFSGS to an infection with HIV is well established and probable for SARS-CoV 2, cases associated with other viral infections are rare and the mechanisms remain incompletely understood due to the limited number of cases. Infections with Parvovirus B19, the cytomegalovirus (CMV), Hepatitis C, simian virus 40 or Epstein-Barr virus are thought to be further potential causes of the development of cFSGS (78). While the CMV seems to be a probable inducer of cFSGS, the association of other viruses and the collapsing variant has still to be proven. Additionally, in 2018 there has been a case series reported the association of dengue virus and zika virus infection and the development of cFSGS (79). In these cases a direct infection of kidney tissue was shown and interestingly, there was no correlation with APOL1 risk alleles. This case study also points to a potential involvement of the complement cascade in the development of the cFSGS. Next to the direct effect of the virus to the podocytes, which has not been shown for these infections, systemic immune response, similar to the response to HIV or SARS-CoV2, might be responsible for upregulation of APOL1 or could directly influence podocyte function (80). A converging mechanism of the innate immune response therefore seems likely, nevertheless has to be further assessed. A summary of these mechanisms is presented in Figure 1.

\section{CONCLUSION}

Research on molecular changes in podocytes expressing risk variants of APOL1 shed light on multiple pathways relevant for 


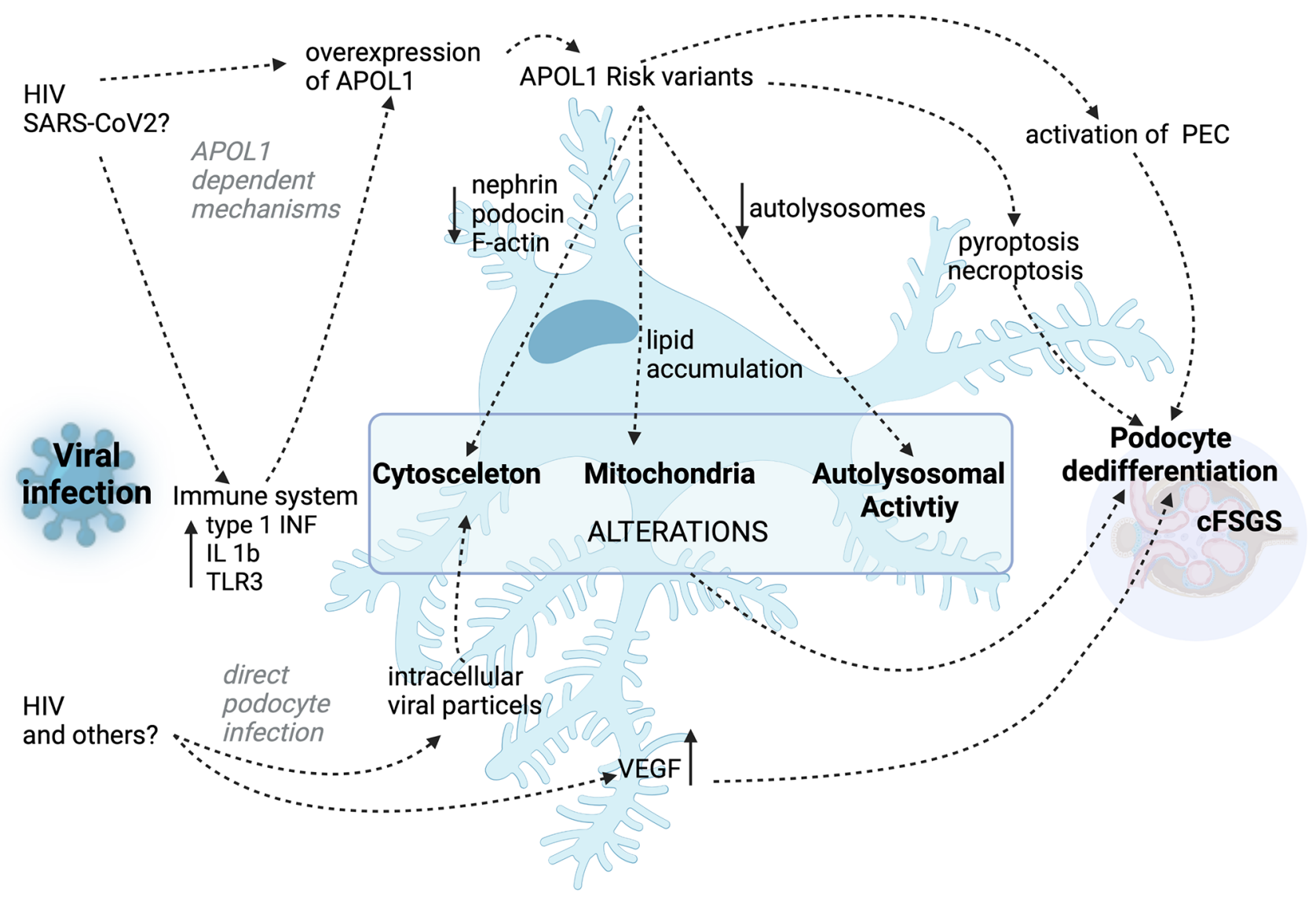

FIGURE 1 | Summary of the proposed APOL1 dependent and APOL1 independent mechanisms in HIV-and other virus-associated cFSGS.

podocyte homeostasis. Although none of the suggested pathways alone could explain the development of cFSGS, the role of a second is evident, at least in APOL1 dependent forms. Limited numbers of patients in case reports hamper the identification of "second hits" like HIV, so the pandemic situation of COVID-19 could help to further scientific effort by providing larger cohorts. Furthermore, the analysis of APOL1-risk factor independent cFSGS forms, the identification of viral etiology and associated damaging mechanisms are crucial to understand the development of the disease. Further investigation of the interaction of viral products and the immune response with podocyte signaling pathways that induce the massive morphologic alterations might contribute to our understanding of podocyte biology and the search for targeted therapies in (collapsing) FSGS independent of its etiology.

\section{REFERENCES}

1. Uffing A, Perez-Saez MJ, Mazzali M, Manfro RC, Bauer AC, de Sottomaior Drumond F, et al. Recurrence of FSGS After Kidney Transplantation in Adults. Clin J Am Soc Nephrol (2020) 15(2):247-56. doi: 10.2215/ CJN.08970719

\section{AUTHOR CONTRIBUTIONS}

AM and SG have performed intensive studies of the current literature and have written main parts of the text and figure. Both first authors contributed equally to this review. TH and FB have performed extensive correction of the text after studying the literature. TH and FB have contributed significantly with the ideas and structure that are found in the review. All authors contributed to the article and approved the submitted version.

\section{FUNDING}

TBH was supported by the DFG (CRC1192), and by the BMBF (STOP-FSGS- 01GM1901C).

2. Kopp JB, Anders HJ, Susztak K, Podesta MA, Remuzzi G, Hildebrandt F, et al. Podocytopathies. Nat Rev Dis Primers (2020) 6(1):68. doi: 10.1038/s41572020-0196-7

3. D'Agati VD, Fogo AB, Bruijn JA, Jennette JC. Pathologic Classification of Focal Segmental Glomerulosclerosis: A Working Proposal. Am J Kidney Dis (2004) 43(2):368-82. doi: 10.1053/j.ajkd.2003.10.024 
4. D'Agati V. Pathologic Classification of Focal Segmental Glomerulosclerosis. Semin Nephrol (2003) 23(2):117-34. doi: 10.1053/snep.2003.50012

5. Mubarak M, Kazi JI. Glomerular Collapse in a Direct Topographic Relationship With Patchy Cortical Infarction in a Native Nephrectomy Specimen. Clin Exp Nephrol (2012) 16(2):360-1. doi: 10.1007/s10157-012-0612-8

6. Bennett AN, Peterson P, Sangle S, Hangartner R, Abbs IC, Hughes GR, et al. Adult Onset Still's Disease and Collapsing Glomerulopathy: Successful Treatment With Intravenous Immunoglobulins and Mycophenolate Mofetil. Rheumatol (Oxford) (2004) 43(6):795-9. doi: 10.1093/rheumatology/keh172

7. Fogo AB, Lusco MA, Najafian B, Alpers CE. AJKD Atlas of Renal Pathology: HIV-Associated Nephropathy (HIVAN). Am J Kidney Dis (2016) 68(2):e13e4. doi: 10.1053/j.ajkd.2016.06.002

8. Nicholas Cossey L, Larsen CP, Liapis H. Collapsing Glomerulopathy: A 30Year Perspective and Single, Large Center Experience. Clin Kidney J (2017) 10 (4):443-9. doi: 10.1093/ckj/sfx029

9. Li SY, Park J, Qiu C, Han SH, Palmer MB, Arany Z, et al. Increasing the Level of Peroxisome Proliferator-Activated Receptor Gamma Coactivator-1alpha in Podocytes Results in Collapsing Glomerulopathy. JCI Insight (2017) 2(14): e92930. doi: 10.1172/jci.insight.92930

10. Cohen AH, Sun NC, Shapshak P, Imagawa DT. Demonstration of Human Immunodeficiency Virus in Renal Epithelium in HIV-Associated Nephropathy. Mod Pathol (1989) 2(2):125-8.

11. Ospina Stella A, Turville S. All-Round Manipulation of the Actin Cytoskeleton by HIV. Viruses (2018) 10(2):63. doi: 10.3390/v10020063

12. Korgaonkar SN, Feng X, Ross MD, Lu TC, D'Agati V, Iyengar R, et al. HIV-1 Upregulates VEGF in Podocytes. J Am Soc Nephrol (2008) 19(5):877-83. doi: 10.1681/ASN.2007050629

13. Veron D, Reidy KJ, Bertuccio C, Teichman J, Villegas G, Jimenez J, et al. Overexpression of VEGF-A in Podocytes of Adult Mice Causes Glomerular Disease. Kidney Int (2010) 77(11):989-99. doi: 10.1038/ki.2010.64

14. Wang H, Misaki T, Taupin V, Eguchi A, Ghosh P, Farquhar MG. GIV/girdin Links Vascular Endothelial Growth Factor Signaling to Akt Survival Signaling in Podocytes Independent of Nephrin. J Am Soc Nephrol (2015) 26(2):314-27. doi: 10.1681/ASN.2013090985

15. Perez-Morga D, Vanhollebeke B, Paturiaux-Hanocq F, Nolan DP, Lins L, Homble F, et al. Apolipoprotein L-I Promotes Trypanosome Lysis by Forming Pores in Lysosomal Membranes. Science (2005) 309(5733):469-72. doi: 10.1126/science. 1114566

16. Freedman BI, Limou S, Ma L, Kopp JB. APOL1-Associated Nephropathy: A Key Contributor to Racial Disparities in CKD. Am J Kidney Dis (2018) $72(5$ Suppl 1):S8-S16. doi: 10.1053/j.ajkd.2018.06.020

17. Cooper A, Ilboudo H, Alibu VP, Ravel S, Enyaru J, Weir W, et al. APOL1 Renal Risk Variants Have Contrasting Resistance and Susceptibility Associations With African Trypanosomiasis. Elife (2017) 6:e25461. doi: 10.7554/eLife.25461

18. Kamoto K, Noyes H, Nambala P, Senga E, Musaya J, Kumwenda B, et al. Association of APOL1 Renal Disease Risk Alleles With Trypanosoma Brucei Rhodesiense Infection Outcomes in the Northern Part of Malawi. PloS Negl Trop Dis (2019) 13(8):e0007603. doi: 10.1371/journal.pntd.0007603

19. Duchateau PN, Pullinger CR, Orellana RE, Kunitake ST, Naya-Vigne J, O'Connor PM, et al. A New Human High Density Lipoprotein Apolipoprotein Expressed by the Pancreas. Identification Cloning Characterization Plasma Distribution Apolipoprotein L. J Biol Chem (1997) 272(41):25576-82. doi: 10.1074/jbc.272.41.25576

20. Madhavan SM, O’Toole JF, Konieczkowski M, Ganesan S, Bruggeman LA, Sedor JR. APOL1 Localization in Normal Kidney and Nondiabetic Kidney Disease. J Am Soc Nephrol (2011) 22(11):2119-28. doi: 10.1681/ASN.2011010069

21. Genovese G, Friedman DJ, Ross MD, Lecordier L, Uzureau P, Freedman BI, et al. Association of Trypanolytic ApoL1 Variants With Kidney Disease in African Americans. Science (2010) 329(5993):841-5. doi: 10.1126/science.1193032

22. Vajgel G, Lima SC, Santana DJS, Oliveira CBL, Costa DMN, Hicks PJ, et al. Effect of a Single Apolipoprotein L1 Gene Nephropathy Variant on the Risk of Advanced Lupus Nephritis in Brazilians. J Rheumatol (2020) 47(8):1209-17. doi: 10.3899/jrheum.190684

23. Kopp JB, Nelson GW, Sampath K, Johnson RC, Genovese G, An P, et al. APOL1 Genetic Variants in Focal Segmental Glomerulosclerosis and HIVAssociated Nephropathy. J Am Soc Nephrol: JASN (2011) 22(11):2129-37. doi: 10.1681/ASN.2011040388
24. Vanhollebeke B, Truc P, Poelvoorde P, Pays A, Joshi PP, Katti R, et al. Human Trypanosoma Evansi Infection Linked to a Lack of Apolipoprotein L-I. N Engl $J$ Med (2006) 355(26):2752-6. doi: 10.1056/NEJMoa063265

25. Lan X, Jhaveri A, Cheng K, Wen H, Saleem MA, Mathieson PW, et al. APOL1 Risk Variants Enhance Podocyte Necrosis Through Compromising Lysosomal Membrane Permeability. Am J Physiol Renal Physiol (2014) 307 (3):F326-36. doi: 10.1152/ajprenal.00647.2013

26. Beckerman P, Bi-Karchin J, Park AS, Qiu C, Dummer PD, Soomro I, et al. Transgenic Expression of Human APOL1 Risk Variants in Podocytes Induces Kidney Disease in Mice. Nat Med (2017) 23(4):429-38. doi: 10.1038/nm.4287

27. Kumar V, Ayasolla K, Jha A, Mishra A, Vashistha H, Lan X, et al. Disrupted Apolipoprotein L1-Mir193a Axis Dedifferentiates Podocytes Through Autophagy Blockade in an APOL1 Risk Milieu. Am J Physiol Cell Physiol (2019) 317(2):C209-C25. doi: 10.1152/ajpcell.00538.2018

28. Wen H, Kumar V, Lan X, Shoshtari SSM, Eng JM, Zhou X, et al. APOL1 Risk Variants Cause Podocytes Injury Through Enhancing Endoplasmic Reticulum Stress. Biosci Rep (2018) 38(4):BSR20171713. doi: 10.1042/BSR20171713

29. Ryu JH, Ge M, Merscher S, Rosenberg AZ, Desante M, Roshanravan H, et al. APOL1 Renal Risk Variants Promote Cholesterol Accumulation in Tissues and Cultured Macrophages From APOL1 Transgenic Mice. PloS One (2019) 14(4):e0211559. doi: 10.1371/journal.pone.0211559

30. Ge M, Molina J, Ducasa GM, Mallela SK, Varona Santos J, Mitrofanova A, et al. APOL1 Risk Variants Affect Podocyte Lipid Homeostasis and Energy Production in Focal Segmental Glomerulosclerosis. Hum Mol Genet (2021) 30 (3-4):182-97. doi: 10.1093/hmg/ddab022

31. Goyal R, Singhal PC. APOL1 Risk Variants and the Development of HIVAssociated Nephropathy. FEBS J (2021) 288(19):5586-97. doi: 10.1111/febs.15677

32. Mikulak J, Oriolo F, Portale F, Tentorio P, Lan X, Saleem MA, et al. Impact of APOL1 Polymorphism and IL-1beta Priming in the Entry and Persistence of HIV-1 in Human Podocytes. Retrovirology (2016) 13(1):63. doi: 10.1186/ s12977-016-0296-3

33. Wakashin H, Heymann J, Roshanravan H, Daneshpajouhnejad P, Rosenberg A, Shin MK, et al. APOL1 Renal Risk Variants Exacerbate Podocyte Injury by Increasing Inflammatory Stress. BMC Nephrol (2020) 21(1):371. doi: 10.1186/ s12882-020-01995-3

34. Nichols B, Jog P, Lee JH, Blackler D, Wilmot M, D'Agati V, et al. Innate Immunity Pathways Regulate the Nephropathy Gene Apolipoprotein L1. Kidney Int (2015) 87(2):332-42. doi: 10.1038/ki.2014.270

35. Schneider WM, Chevillotte MD, Rice CM. Interferon-Stimulated Genes: A Complex Web of Host Defenses. Annu Rev Immunol (2014) 32:513-45. doi: 10.1146/annurev-immunol-032713-120231

36. Doyle T, Goujon C, Malim MH. HIV-1 and Interferons: Who's Interfering With Whom? Nat Rev Microbiol (2015) 13(7):403-13. doi: 10.1038/nrmicro3449

37. Markowitz GS, Nasr SH, Stokes MB, D'Agati VD. Treatment With IFN\{Alpha\}, -\{Beta\}, or -\{Gamma\} Is Associated With Collapsing Focal Segmental Glomerulosclerosis. Clin J Am Soc Nephrol (2010) 5(4):607-15. doi: 10.2215/ CJN.07311009

38. Aghajan M, Booten SL, Althage M, Hart CE, Ericsson A, Maxvall I, et al. Antisense Oligonucleotide Treatment Ameliorates IFN-Gamma-Induced Proteinuria in APOL1-Transgenic Mice. JCI Insight (2019) 4(12):e126124. doi: $10.1172 /$ jci.insight. 126124

39. Gao D, Wu J, Wu YT, Du F, Aroh C, Yan N, et al. Cyclic GMP-AMP Synthase Is an Innate Immune Sensor of HIV and Other Retroviruses. Science (2013) 341(6148):903-6. doi: 10.1126/science. 1240933

40. Jakobsen MR, Bak RO, Andersen A, Berg RK, Jensen SB, Tengchuan J, et al. IFI16 Senses DNA Forms of the Lentiviral Replication Cycle and Controls HIV-1 Replication. Proc Natl Acad Sci USA (2013) 110(48):E4571-80. doi: 10.1073/pnas.1311669110

41. Davis SE, Khatua AK, Popik W. Nucleosomal dsDNA Stimulates APOL1 Expression in Human Cultured Podocytes by Activating the cGAS/IFI16STING Signaling Pathway. Sci Rep (2019) 9(1):15485. doi: 10.1038/s41598019-51998-w

42. Wu J, Ma Z, Raman A, Beckerman P, Dhillon P, Mukhi D, et al. APOL1 Risk Variants in Individuals of African Genetic Ancestry Drive Endothelial Cell Defects That Exacerbate Sepsis. Immunity (2021) 54(11):2632-49.e6. doi: 10.1016/j.immuni.2021.10.004

43. Kato Y, Park J, Takamatsu H, Konaka H, Aoki W, Aburaya S, et al. ApoptosisDerived Membrane Vesicles Drive the cGAS-STING Pathway and Enhance 
Type I IFN Production in Systemic Lupus Erythematosus. Ann Rheum Dis (2018) 77(10):1507-15. doi: 10.1136/annrheumdis-2018-212988

44. Fang J, Yao X, Hou M, Duan M, Xing L, Huang J, et al. ApoL1 Induces Kidney Inflammation Through RIG-I/NF-kappaB Activation. Biochem Biophys Res Commun (2020) 527(2):466-73. doi: 10.1016/j.bbrc.2020.04.054

45. Appel D, Kershaw DB, Smeets B, Yuan G, Fuss A, Frye B, et al. Recruitment of Podocytes From Glomerular Parietal Epithelial Cells. J Am Soc Nephrol (2009) 20(2):333-43. doi: 10.1681/ASN.2008070795

46. Sagrinati C, Netti GS, Mazzinghi B, Lazzeri E, Liotta F, Frosali F, et al. Isolation and Characterization of Multipotent Progenitor Cells From the Bowman's Capsule of Adult Human Kidneys. J Am Soc Nephrol (2006) 17 (9):2443-56. doi: 10.1681/ASN.2006010089

47. Wanner N, Hartleben B, Herbach N, Goedel M, Stickel N, Zeiser R, et al. Unraveling the Role of Podocyte Turnover in Glomerular Aging and Injury. J Am Soc Nephrol (2014) 25(4):707-16. doi: 10.1681/ASN.2013050452

48. Puelles VG, van der Wolde JW, Wanner N, Scheppach MW, Cullen-McEwen LA, Bork T, et al. mTOR-Mediated Podocyte Hypertrophy Regulates Glomerular Integrity in Mice and Humans. JCI Insight (2019) 4(18):e99271. doi: 10.1172/jci.insight. 99271

49. Kumar V, Vashistha H, Lan X, Chandel N, Ayasolla K, Shoshtari SSM, et al. Role of Apolipoprotein L1 in Human Parietal Epithelial Cell Transition. Am J Pathol (2018) 188(11):2508-28. doi: 10.1016/j.ajpath.2018.07.025

50. Merchant ML, Barati MT, Caster DJ, Hata JL, Hobeika L, Coventry S, et al. Proteomic Analysis Identifies Distinct Glomerular Extracellular Matrix in Collapsing Focal Segmental Glomerulosclerosis. J Am Soc Nephrol (2020) 31 (8):1883-904. doi: 10.1681/ASN.2019070696

51. Smeets B, Stucker F, Wetzels J, Brocheriou I, Ronco P, Grone HJ, et al. Detection of Activated Parietal Epithelial Cells on the Glomerular Tuft Distinguishes Early Focal Segmental Glomerulosclerosis From Minimal Change Disease. Am J Pathol (2014) 184(12):3239-48. doi: 10.1016/j.ajpath.2014.08.007

52. Cheng $\mathrm{Y}$, Luo R, Wang $\mathrm{X}$, Wang $\mathrm{K}$, Zhang $\mathrm{N}$, Zhang $\mathrm{M}$, et al. The Incidence, Risk Factors, and Prognosis of Acute Kidney Injury in Adult Patients With Coronavirus Disease 2019. Clin J Am Soc Nephrol (2020) 15(10):1394-402. doi: 10.2215/CJN.04650420

53. Cheng Y, Luo R, Wang K, Zhang M, Wang Z, Dong L, et al. Kidney Disease Is Associated With in-Hospital Death of Patients With COVID-19. Kidney Int (2020) 97(5):829-38. doi: 10.1016/j.kint.2020.03.005

54. Hirsch JS, Ng JH, Ross DW, Sharma P, Shah HH, Barnett RL, et al. Acute Kidney Injury in Patients Hospitalized With COVID-19. Kidney Int (2020) 98 (1):209-18. doi: 10.1016/j.kint.2020.05.006

55. Naicker S, Yang CW, Hwang SJ, Liu BC, Chen JH, Jha V. The Novel Coronavirus 2019 Epidemic and Kidneys. Kidney Int (2020) 97(5):824-8. doi: 10.1016/j.kint.2020.03.001

56. Su H, Yang M, Wan C, Yi LX, Tang F, Zhu HY, et al. Renal Histopathological Analysis of 26 Postmortem Findings of Patients With COVID-19 in China. Kidney Int (2020) 98(1):219-27. doi: 10.1016/j.kint.2020.04.003

57. Oliveira EA, Oliveira MCL, Martelli DB, Colosimo EA, Silva LR, Lanza K, et al. COVID-19 Pandemic and the Answer of Science: A Year in Review. Acad Bras Cienc (2021) 93(4):e20210543. doi: 10.1590/0001-3765202120210543

58. Le Stang MB, Desenclos J, Flamant M, Chousterman BG, Tabibzadeh N. The Good Treatment, the Bad Virus, and the Ugly Inflammation: Pathophysiology of Kidney Involvement During COVID-19. Front Physiol (2021) 12:613019. doi: 10.3389/fphys.2021.613019

59. Velez JCQ, Caza T, Larsen CP. COVAN Is the New HIVAN: The ReEmergence of Collapsing Glomerulopathy With COVID-19. Nat Rev Nephrol (2020) 16(10):565-7. doi: 10.1038/s41581-020-0332-3

60. Laboux T, Gibier JB, Pottier N, Glowacki F, Hamroun A. COVID-19-Related Collapsing Glomerulopathy Revealing a Rare Risk Variant of APOL1: Lessons for the Clinical Nephrologist. J Nephrol (2021) 34(2):373-8. doi: 10.1007/ s40620-020-00935-6

61. Magoon S, Bichu P, Malhotra V, Alhashimi F, Hu Y, Khanna S, et al. COVID19-Related Glomerulopathy: A Report of 2 Cases of Collapsing Focal Segmental Glomerulosclerosis. Kidney Med (2020) 2(4):488-92. doi: 10.1016/j.xkme.2020.05.004

62. Lazareth H, Pere H, Binois Y, Chabannes M, Schurder J, Bruneau T, et al. COVID-19-Related Collapsing Glomerulopathy in a Kidney Transplant Recipient. Am J Kidney Dis (2020) 76(4):590-4. doi: 10.1053/ j.ajkd.2020.06.009
63. Batlle D, Soler MJ, Sparks MA, Hiremath S, South AM, Welling PA, et al. Acute Kidney Injury in COVID-19: Emerging Evidence of a Distinct Pathophysiology. J Am Soc Nephrol (2020) 31(7):1380-3. doi: 10.1681/ ASN.2020040419

64. Puelles VG, Lutgehetmann M, Lindenmeyer MT, Sperhake JP, Wong MN, Allweiss L, et al. Multiorgan and Renal Tropism of SARS-CoV-2. N Engl J Med (2020) 383(6):590-2. doi: 10.1056/NEJMc2011400

65. Bradley BT, Maioli H, Johnston R, Chaudhry I, Fink SL, Xu H, et al. Histopathology and Ultrastructural Findings of Fatal COVID-19 Infections in Washington State: A Case Series. Lancet (2020) 396(10247):320-32. doi: 10.1016/S0140-6736(20)31305-2

66. Braun F, Lutgehetmann M, Pfefferle S, Wong MN, Carsten A, Lindenmeyer MT, et al. SARS-CoV-2 Renal Tropism Associates With Acute Kidney Injury. Lancet (2020) 396(10251):597-8. doi: 10.1016/S0140-6736(20)31759-1

67. Peleg Y, Kudose S, D'Agati V, Siddall E, Ahmad S, Nickolas T, et al. Acute Kidney Injury Due to Collapsing Glomerulopathy Following COVID-19 Infection. Kidney Int Rep (2020) 5(6):940-5. doi: 10.1016/j.ekir.2020.04.017

68. Dorward DA, Russell CD, Um IH, Elshani M, Armstrong SD, Penrice-Randal $\mathrm{R}$, et al. Tissue-Specific Immunopathology in Fatal COVID-19. Am J Respir Crit Care Med (2021) 203(2):192-201. doi: 10.1164/rccm.202008-3265OC

69. Braun F, Edler C, Puelles VG, Huber TB. Association of SARS-CoV-2 Renal Tropism With Acute Kidney Injury - Authors' Reply. Lancet (2020) 396 (10266):1881-2. doi: 10.1016/S0140-6736(20)32586-1

70. Diao B, Wang C, Wang R, Feng Z, Zhang J, Yang H, et al. Human Kidney Is a Target for Novel Severe Acute Respiratory Syndrome Coronavirus 2 Infection. Nat Commun (2021) 12(1):2506. doi: 10.1038/s41467-021-22781-1

71. Legrand M, Bell S, Forni L, Joannidis M, Koyner JL, Liu K, et al. Pathophysiology of COVID-19-Associated Acute Kidney Injury. Nat Rev Nephrol (2021) 17(11):751-64. doi: 10.1038/s41581-021-00452-0

72. Huang C, Wang Y, Li X, Ren L, Zhao J, Hu Y, et al. Clinical Features of Patients Infected With 2019 Novel Coronavirus in Wuhan, China. Lancet (2020) 395(10223):497-506. doi: 10.1016/S0140-6736(20)30183-5

73. Liu K, Yang T, Peng XF, Lv SM, Ye XL, Zhao TS, et al. A Systematic MetaAnalysis of Immune Signatures in Patients With COVID-19. Rev Med Virol (2021) 31(4):e2195. doi: 10.1002/rmv.2195

74. Sun YB, Qu X, Zhang X, Caruana G, Bertram JF, Li J. Glomerular Endothelial Cell Injury and Damage Precedes That of Podocytes in Adriamycin-Induced Nephropathy. PloS One (2013) 8(1):e55027. doi: 10.1371/journal.pone.0055027

75. Zhang J, Hanig JP, De Felice AF. Biomarkers of Endothelial Cell Activation: Candidate Markers for Drug-Induced Vasculitis in Patients or Drug-Induced Vascular Injury in Animals. Vascul Pharmacol (2012) 56(1-2):14-25. doi: 10.1016/j.vph.2011.09.002

76. Wu H, Larsen CP, Hernandez-Arroyo CF, Mohamed MMB, Caza T, Sharshir $\mathrm{M}$, et al. AKI and Collapsing Glomerulopathy Associated With COVID-19 and APOL 1 High-Risk Genotype. J Am Soc Nephrol (2020) 31(8):1688-95. doi: 10.1681/ASN.2020050558

77. Ahmadian E, Hosseiniyan Khatibi SM, Razi Soofiyani S, Abediazar S, Shoja MM, Ardalan M, et al. Covid-19 and Kidney Injury: Pathophysiology and Molecular Mechanisms. Rev Med Virol (2021) 31(3):e2176. doi: 10.1002/ rmv. 2176

78. Chandra P, Kopp JB. Viruses and Collapsing Glomerulopathy: A Brief Critical Review. Clin Kidney J (2013) 6(1):1-5. doi: 10.1093/ckj/sft002

79. Araujo SA, Cordeiro TME, Belisario AR, Araujo RFA, Marinho PES, Kroon EG, et al. First Report of Collapsing Variant of Focal Segmental Glomerulosclerosis Triggered by Arbovirus: Dengue and Zika Virus Infection. Clin Kidney J (2019) 12(3):355-61. doi: 10.1093/ckj/sfy104

80. Zheng H, Rao AM, Dermadi D, Toh J, Murphy Jones L, Donato M, et al. Multi-Cohort Analysis of Host Immune Response Identifies Conserved Protective and Detrimental Modules Associated With Severity Across Viruses. Immunity (2021) 54(4):753-68.e5. doi: 10.1016/j.immuni.2021.03.002

Conflict of Interest: The authors declare that the research was conducted in the absence of any commercial or financial relationships that could be construed as a potential conflict of interest.

Publisher's Note: All claims expressed in this article are solely those of the authors and do not necessarily represent those of their affiliated organizations, or those of the publisher, the editors and the reviewers. Any product that may be evaluated in 
this article, or claim that may be made by its manufacturer, is not guaranteed or endorsed by the publisher.

Copyright $\odot 2022$ Muehlig, Gies, Huber and Braun. This is an open-access article distributed under the terms of the Creative Commons Attribution License
(CC BY). The use, distribution or reproduction in other forums is permitted, provided the original author(s) and the copyright owner(s) are credited and that the original publication in this journal is cited, in accordance with accepted academic practice. No use, distribution or reproduction is permitted which does not comply with these terms. 